TITLE:

\title{
Cluster species and cluster size dependence of damage formation by cluster ion impact
}

$\operatorname{AUTHOR}(\mathrm{S}):$

Aoki, Takaaki; Matsuo, Jiro; Takaoka, Gikan; Yamada, Isao

\section{CITATION:}

Aoki, Takaaki ... [et al]. Cluster species and cluster size dependence of damage formation by cluster ion impact. Nuclear Instruments and Methods in Physics Research Section B: Beam Interactions with Materials and Atoms 2003, 206: 861-865

ISSUE DATE:

2003-05

URL:

http://hdl.handle.net/2433/8944

\section{RIGHT:}

この論文は出版社版でありません。引用の際には出版社版をご確認ご 利用ください。; This is not the published version. Please cite only the published version. 
Cluster species and cluster size dependence of damage formation by cluster ion impact.

Takaaki Aoki $^{1,3)}$, Jiro Matsuo ${ }^{1)}$, Gikan Takaoka ${ }^{1)}$ and Isao Yamada ${ }^{2,3)}$

1) Ion Beam Engineering Experimental Laboratory, Kyoto University, Sakyo, Kyoto 606-8501, JAPAN

2) Laboratory of Advanced Science and Technology for Industry, Himeji Institute of Technology, CAST, 3-1-2 Kouto, Kamigori-cho, Ako-gun, Hyogo, 678-1205 JAPAN

3) Collaborative Research Center for Cluster Ion Beam Process Technology.

\section{$\underline{\text { ABSTRACT }}$}

Molecular dynamics simulations of $\mathrm{Ne}, \mathrm{Ar}$ and Xe clusters with various sizes impacting on $\mathrm{Si}$ surfaces were performed in order to study damage formation processes. When cluster size ranged from several tens to several thousands and accelerated energy is $20 \mathrm{keV}$ in total, each cluster impact caused surface damage in the shape of a crater. A larger number of displacements was found with the Xe cluster than Ne and Ar clusters of the same cluster size because the Xe cluster had a larger mass and momentum. However, when cluster size exceeded several thousands, different surface damage structures were formed depending on the cluster species. At a cluster size of 10,000, for example, a crater-like trace was still formed with a $\mathrm{Ne}$ cluster impact. However, both the depth of the crater and the number of displacements decreased as the atomic number of the cluster atom increased, which meant that the atomic and energy density irradiated on the surface is a dominant parameter in causing surface damage. These results indicate that the damage formation by cluster impact can be controlled by 
changing the cluster species as well as the incident energy and cluster size.

PACS: 61.80.Lj, 36.40.-c,45.50.-j

Keywords: Cluster impact, Molecular Dynamics, Damage Formation, Rare Gas Cluster

Corresponding Author:

Takaaki Aoki

Ion Beam Engineering Experimental Laboratory, Kyoto University

Sakyo Kyoto, 606-8501, JAPAN

TEL: +81-75-753-4994 / FAX: +81-75-751-6774

e-mail: t-aoki@kuee.kyoto-u.ac.jp

\section{$\underline{\text { INTRODUCTION }}$}

Cluster ion beam irradiation provides characteristic and superior surface modification processes compared to those using the usual monomer ion beams. The atomic-level surface smoothing and high-sputtering yield are demonstrated by Ar cluster ion irradiation with a size (number of atoms in a cluster) of several thousands and the accelerated energy of several-tens $\mathrm{keV}$ [1]. Recently, these technologies have started to be applied to industrial processes of nano-scale surface treatments [1-3]. The irradiation effects of cluster ions depend on various irradiation parameters; accelerated energy, cluster size, cluster species and so on. In order to realize high-performance surface modification processes with cluster ion beams, these parameters should be controlled according to each suggested process. Computer simulation is a useful method of studying the impact processes of cluster ions and is expected to predict the 
best irradiation conditions. In this paper, we studied the collisional processes of clusters of various sizes and species using molecular dynamics simulations. We examined how variations of these parameters affect damage formation.

\section{$\underline{\text { SIMULATION MODEL }}$}

In this study, the molecular dynamics (MD) method was applied in order to examine the collisional processes of rare-gas clusters onto silicon substrates. The detail of our MD simulation method is shown in previous works [4]. Ne, Ar, and Xe clusters with totally $20 \mathrm{keV}$ of incident energy were irradiated on an Si target. The cluster size ranged from 20 to 20,000, which corresponds to the size distribution of Ar clusters in real experiments [5]. Before impact, each cluster had a highly-packed-cubic (hpc) structure and atomic distance was set to agree with its density in the solid state. A large Si (001) target consisting of about 2,000,000 atoms with the dimensions of $400 \AA \times 400 \AA \times 200 \AA$ was prepared. The MD simulations were performed for $16 \mathrm{ps}$, which is long enough to observe the impact process of the cluster and solid target, for example, the penetration and re-evaporation of the cluster atom, and formation and relaxation of displaced target atoms.

\section{RESULTS AND DISCUSSION}

Figure 1 shows snapshots of $\mathrm{Ne}, \mathrm{Ar}$, and Xe clusters with the size of 2,000 impacting on a Si (001) surface, 16ps after the impact. Each snapshot is drawn from sliced substrate of $40 \AA$ thickness including the impact point. The total energy of each cluster is $20 \mathrm{keV}$, so that 
each atom in the clusters is accelerated at $10 \mathrm{eV}$. Black and white circles indicate incident rare-gas atoms and target atoms, respectively. Large white circles represent displaced target atoms, which are defined as atoms that are below $2.5 \AA$ from the surface level and with potential energies above $0.2 \mathrm{eV}$ from bulk status [6]. Figure 1 shows that, in each case of impact, the cluster caused a large number of displacements and a crater-like surface structure. The dimensions of craters formed by $\mathrm{Ne}, \mathrm{Ar}$, and Xe clusters are similar to each other. However, the distributions of displacements surrounding the craters are quite different. The Xe cluster impact causes a noticeably deeper distribution than Ar and Ne cluster impacts. Presumably, this is due to the fact that the heavier Xe cluster carries a larger momentum, so that formation of displacements is more likely compared to Ar and Ne clusters.

When cluster size increases, the structure of the damage becomes different depending on the cluster species. Figure 2 shows snapshots of Ne, Ar, and Xe cluster impacts with a cluster size of 10,000 . In Fig. 2, total incident energy is kept at $20 \mathrm{keV}$, but the structures of irradiated surfaces are quite different, and species dependence is more obvious compared with Fig. 1. A crater-like structure is shown only with Ne cluster impact. On the other hand, for Ar and Xe cluster impacts, the cluster does not penetrate the surface and breaks up on the target. The surface of the target that was bent downward by the cluster impact recovers immediately after the corruption and evaporation of the cluster, and remains flat.

Figure 3 shows the cluster size dependence of a number of displacements. For each species of cluster, the number of displacements initially increases with cluster size but de- 
creases for very large cluster sizes. As the atomic number of cluster atoms increases, the curve shifts to the left. It is considered that the shift of the curve is due to the differences in momentum and density of particles among these clusters. Figure 4 shows the dependence of the number of displacements on the total momentum of the irradiated cluster. Momentum is calculated by $\left(N \times m \times E_{\mathrm{k}}\right)^{1 / 2}$, where $N, m$ and $E_{\mathrm{k}}$ represent cluster size, mass of constituent atom, and total kinetic energy of cluster, respectively. It is clear that the increase in momentum could be due to the increase in either cluster size or atomic mass in the cluster. As shown in Fig. 4, the curves in the low momentum region show good similarity independent of the cluster species, while behaviors in the higher momentum region are different. The lighter atomic cluster shows a rapid decline at lower momentum.

Figure 5 shows the number of displacements as a function of incident energy density. The incident energy is calculated by dividing the total incident energy by a cross-section of the cluster ( $=4 \pi r^{2} ; r$ : radius of cluster). When energy density is below $4 \mathrm{eV} / \AA^{2}$, the number of displacements rapidly decreases and no damage is caused below $2 \mathrm{eV} / \AA^{2}$. This threshold energy density is considered as the least energy density for a large, low-energy cluster to penetrate the surface by the clearing-way effect [7]. With this energy density, the total deposited energy in one lattice unit area of $\mathrm{Si}(100)$ surface $(5.4 \AA \times 5.4 \AA)$ is calculated as about $55 \mathrm{eV}$. It is estimated that 5 6 surface $\mathrm{Si}$ atoms can be displaced, considering the defect formation energy for a Si atom in the bulk to be about $10 \mathrm{eV}$. In this work, the displacement is defined as an atom residing 2 monolayers below the surface. Therefore, it is concluded that the above mentioned threshold energy density is acceptable. 


\section{SUMMARY}

The damage formation processes by $\mathrm{Ne}$, Ar, and Xe cluster ion impacts were studied using molecular dynamics simulations. When the total energy was constant, the number of displacements depended on both the size and species of the cluster. With a cluster size ranging from several tens to several thousands, a cluster could penetrate the surface and many displacements were generated in a crater-like structure. When the cluster size was kept the same, an Xe cluster caused a larger number of displacements than an $\mathrm{Ne}$ or Ar cluster, since the Xe cluster consisted of heavier atoms and thus had higher momentum. In this cluster size regime, a similar number of displacements could be normalized by the momenta of the cluster independently of the cluster species. For cluster sizes over several thousands, the number of displacements decreased as the cluster size increased. This is because of the reduction of energy deposition density due to the increase of cluster size and cluster volume. As the energy density decreased, it became more difficult for cluster atoms to knock on the surface atom and to impinge into the surface through multiple-collisions. The threshold energy density to cause damage was found as $2 \mathrm{eV} / \AA^{2}$, which is comparable to the value that causes several displacements per one lattice unit. These results indicate that damage formation by cluster impact can be controlled by changing the cluster species, as well as the incident energy and cluster size.

\section{ACKNOWLEDGEMENT}

The authors would like to acknowledge NEDO (New energy and industrial technology de- 
velopment organization) in Japan for supporting this work.

\section{REFERENCES}

[1] I. Yamada, J. Matsuo, Z. Insepov, T. Aoki, T. Seki and N. Toyoda, Nucl. Instr. and Meth., B 164-165 (2000) 944.

[2] I. Yamada, T. Kitagawa, J. Matsuo and A. Kirkpatrick, Mass and charge transport in inorganic materials: fundamentals to devices, part B (Advances in science and technology 29), (2000) pp. 957,

[3] J. Matsuo, H. Katsumata, E. Minami and I. Yamada, Nucl. Instr. and Meth., B 161-163 (2000) 952 .

[4] T. Aoki, J. Matsuo and G. Takaoka, "Molecular Dynamics Study of Damage Formation Characteristics by Large Cluster Ion Impacts", Nucl. Instr. and Meth. B, in publication and to be published in 2003 .

[5] T. Seki, J. Matsuo, G. H. Takaoka, I. Yamada, "Generation of the Large Current Cluster Ion Beam", in this issue.

[6] M. J. Catula, T. diaz de la Rubia and G. H. Gilmer, Nucl. Instr. and Meth., B 196 (1995) 1.

[7] V. I. Shulga and P. Sigmund, Nucl. Instr. and Meth., B47 (1990) 236 


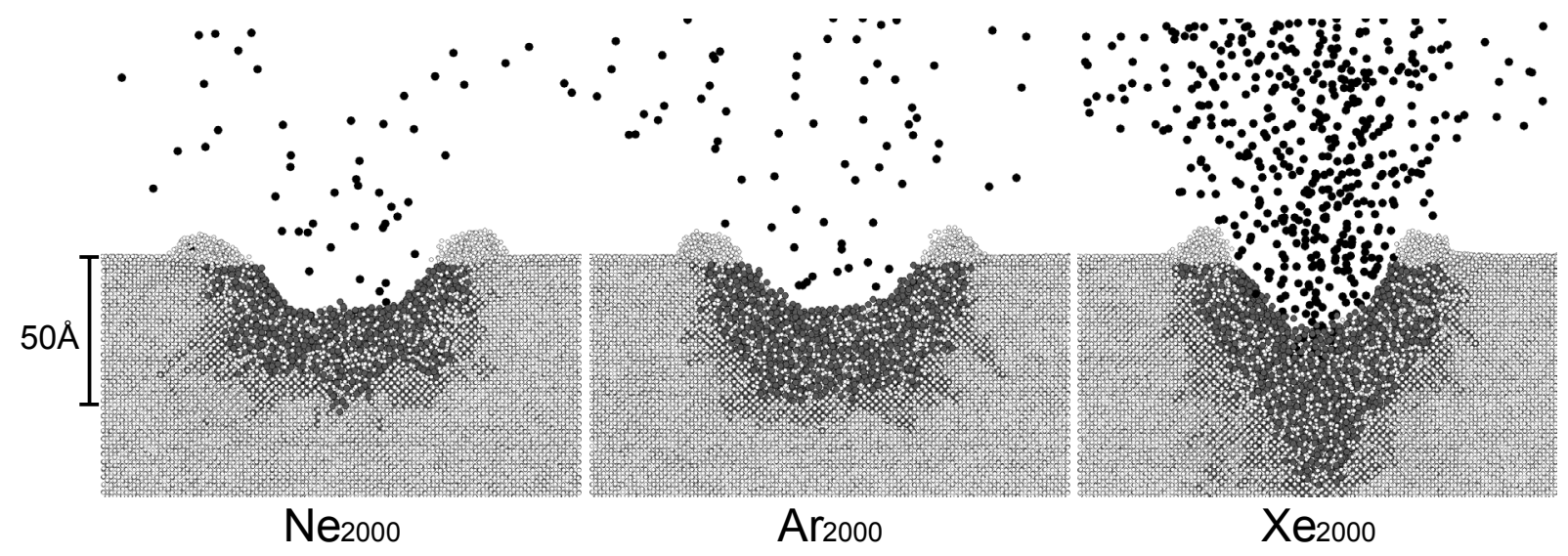

Figure 1: Snapshots of Ne, Ar, and Xe clusters impacting on Si (100) surfaces with a size of 2,000 and total energy of $20 \mathrm{keV}, 16 \mathrm{ps}$ after impact.

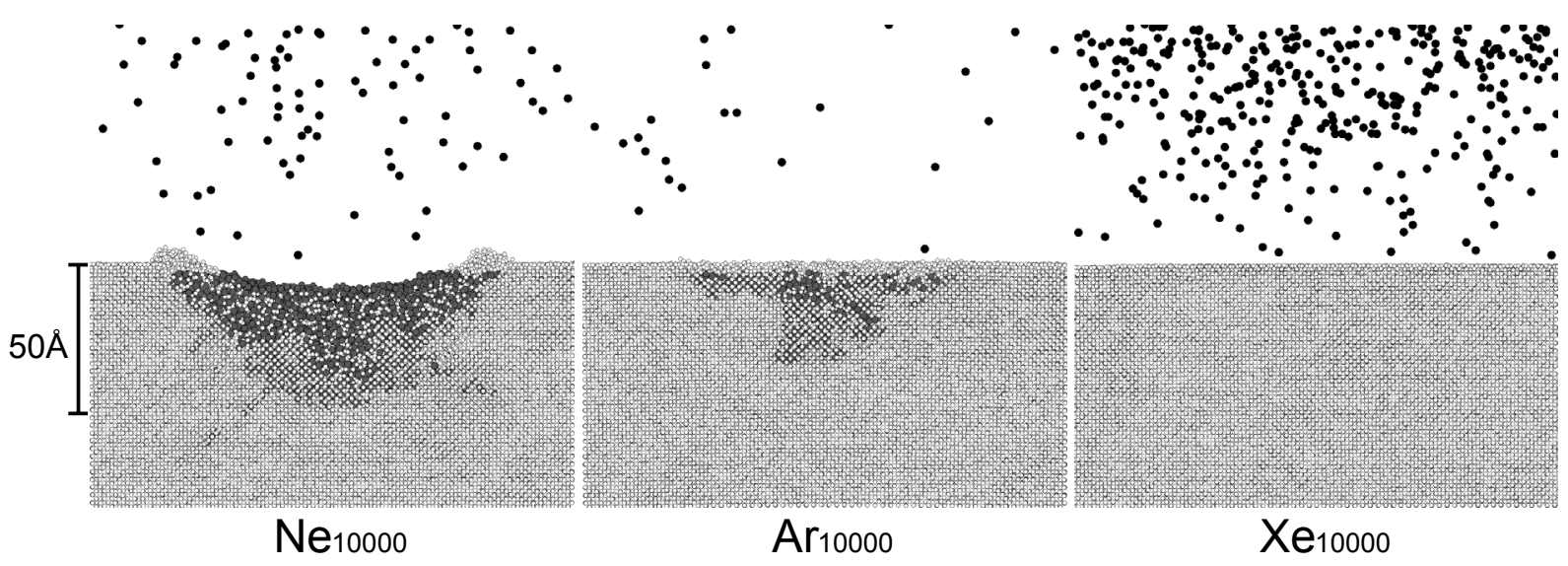

Figure 2: Snapshots of $\mathrm{Ne}$, Ar, and Xe clusters impacting on Si (100) surfaces with size of 10,000 and total energy of $20 \mathrm{keV}, 16 \mathrm{ps}$ after impact. 


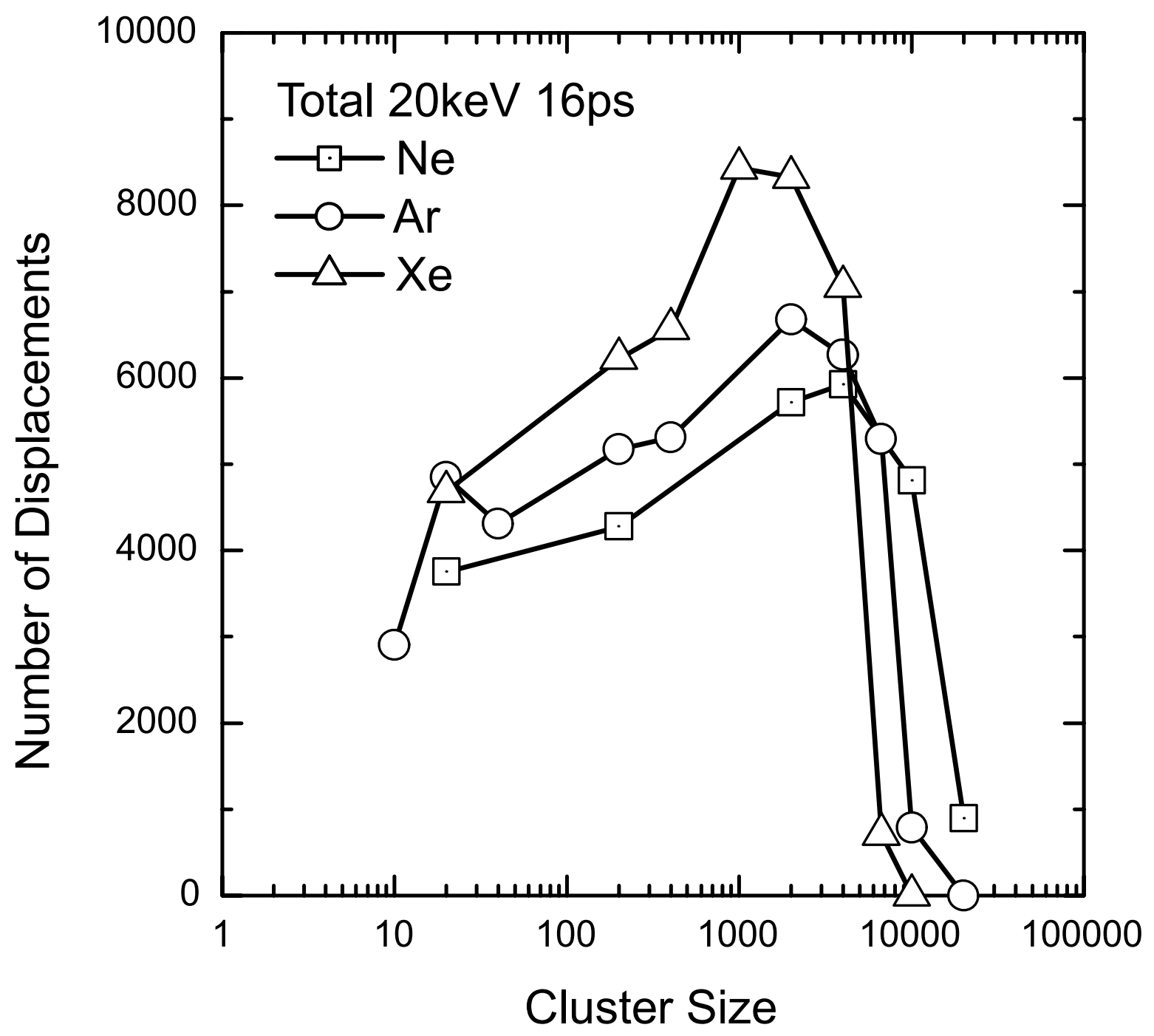

Figure 3: Cluster size dependence of a number of displacements at impacts of clusters of various species. Displacement is defined as an atom of which the potential energy is above $0.2 \mathrm{eV}$ from bulk status. 


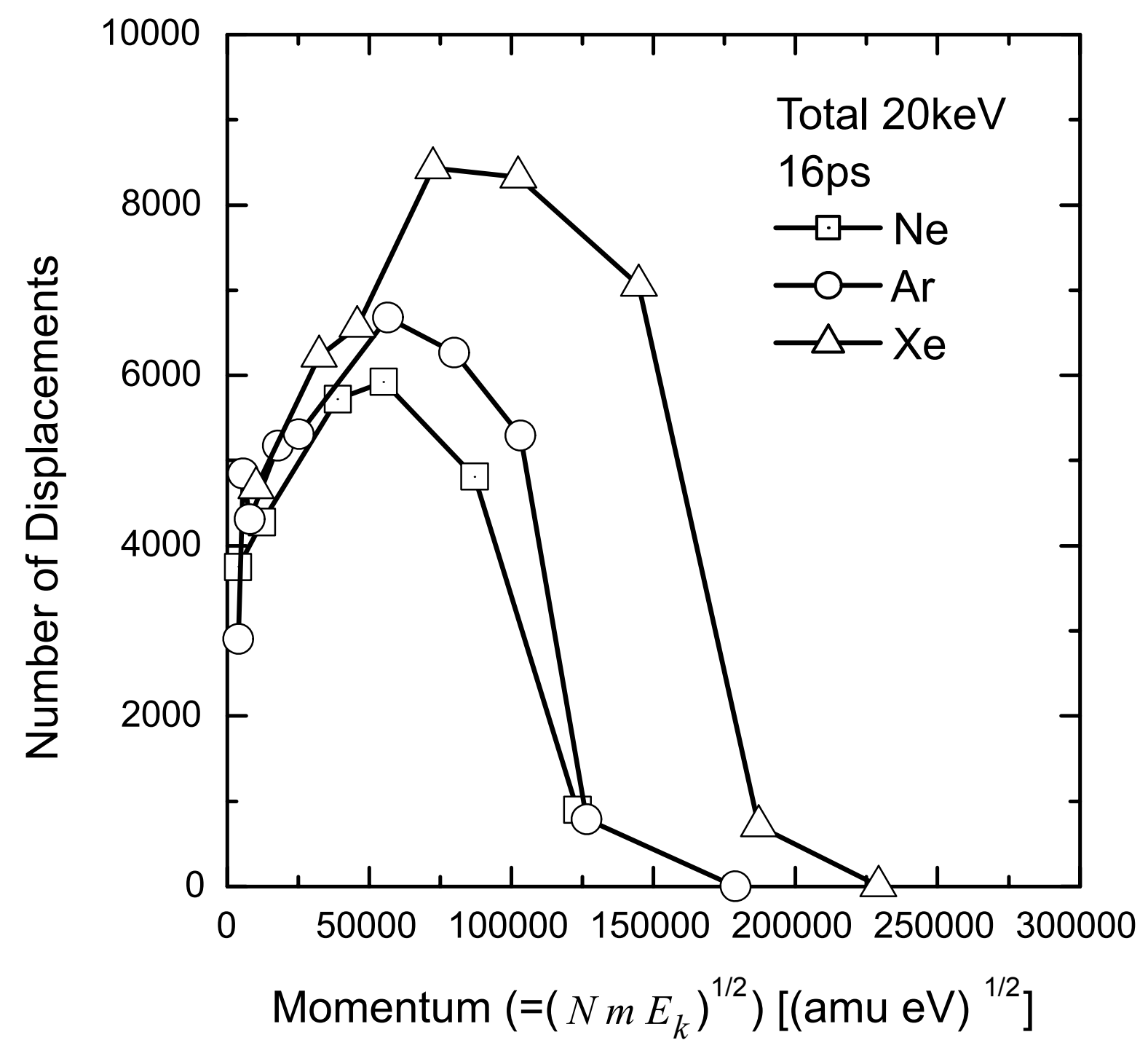

Figure 4: Dependence of a number of displacements as the function of momentum of each cluster. 


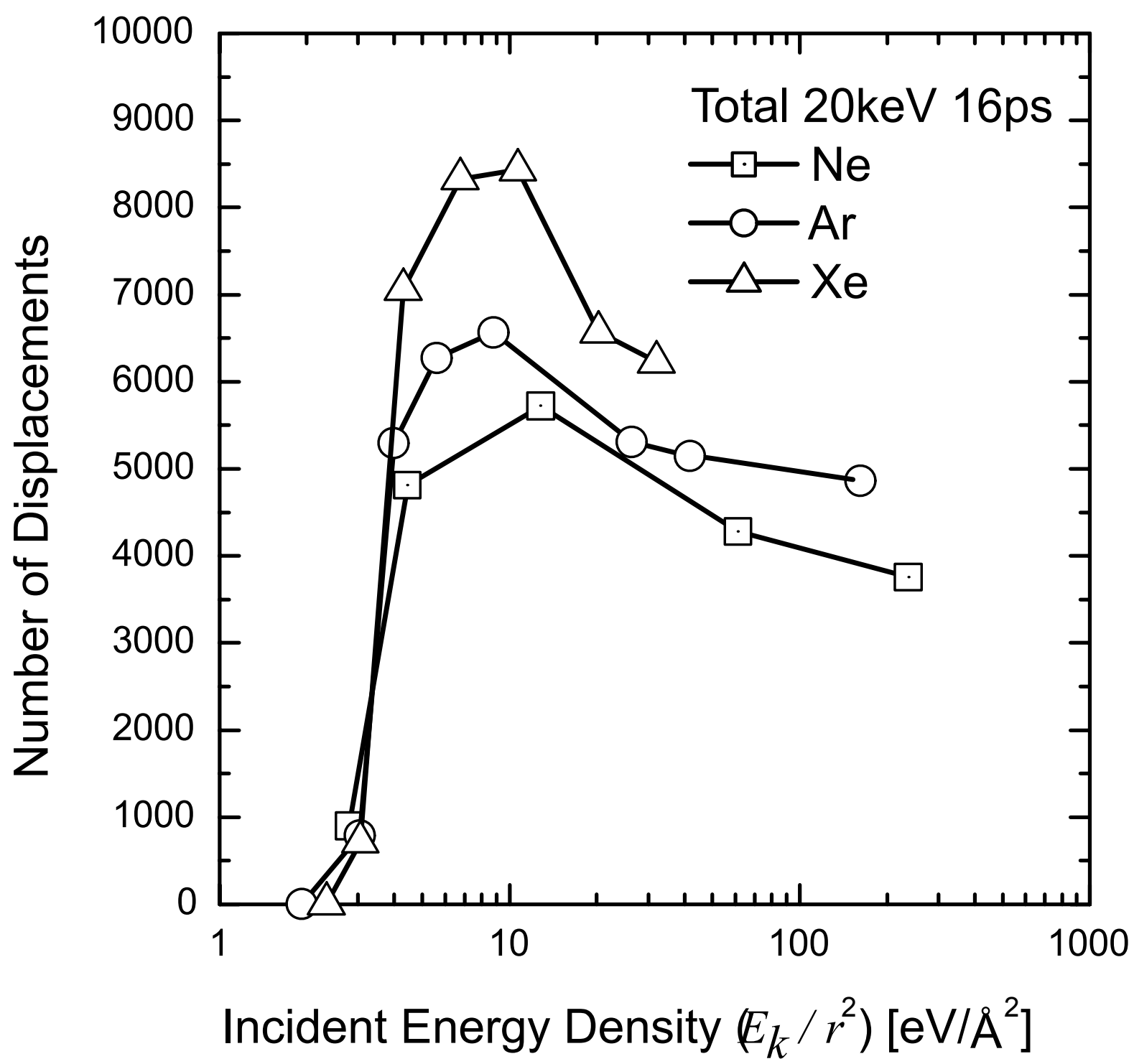

Figure 5: Dependence of a number of displacements as the function of energy density, calculated by dividing total energy by area of cross-section of clusters. 